\title{
Study on Anomalous Electron Diffusion in the Hall Effect Thruster
}

\author{
Kybeom Kwon* \\ Department of Aerospace Engineering, Air Force Academy, Chungbuk, 363-849, Republic of Korea
}

\author{
Mitchell L. R. Walker** and Dimitri N. Mavris*** \\ Department of Aerospace Engineering, Georgia Institute of Technology, Atlanta, GA 30332, USA
}

\begin{abstract}
Over the last two decades, numerous experimental and numerical efforts have examined physical phenomena in plasma discharge devices. The physical mechanisms that govern the anomalous electron diffusion from the cathode to the anode in the Hall Effect Thruster (HET) are not fully understood. This work used 1-D numerical method to improve our understanding and gain insight into the effect of the anomalous electron diffusion in the HET. To this end, numerical solutions are compared with various experimental HET performance measurements and the effects of anomalous electron diffusion are analyzed. The relationships between the anomalous electron diffusion and important parameters of the HET are also studied quantitatively. The work identifies the cathode mass flow rate fraction, radial magnetic field distribution, and discharge voltage as significant factors that affect anomalous electron diffusion. Additionally, the study demonstrates a computational process to determine the radial magnetic field distribution required to achieve specific thruster performance goals.
\end{abstract}

Key words: Hall effect thruster, anomalous electron diffusion, statistical approach

\section{Introduction}

The HET is a type of plasma discharge devices used for space propulsion [1]. It uses electrical energy to ionize and accelerate gas (usually xenon) to produce thrust. Figure 1 shows a schematic of a typical HET. A metallic plate located in the rear of the annular discharge channel serves as the anode and propellant gas distributor. Electrons from the cathode are attracted to the positively-biased anode. To increase the electrons residence time in the discharge channel, the HET employs a radial magnetic field that magnetizes the electrons, but not ions. The radial magnetic and axial electric field (potential difference between the anode and the downstream plasma potential) generate an azimuthal electron current $(\mathrm{E} \times \mathrm{B}$ drift, where $\mathrm{E}$ is the electric field strength and $\mathrm{B}$ is the magnetic field strength), which is called the Hall current. The electrons in the Hall current collide with the neutral propellant to produce ions. The ions accelerate out of the thruster due to the axial electric field, which produces thrust. To complete the electrical circuit in the HET, magnetized electrons must diffuse across the radial magnetic field lines to the anode.

Classical theory assumes that electron conductivity across a magnetic field is governed by electron-neutral and Coulomb collisions. However, these collisions are insufficient to explain the experimentally observed electron transport [2]. Anomalous electron diffusion is a controversial topic among HET researchers and after more than 50 years of investigation it is still not fully understood. Some work attributes the anomalous electron diffusion to electron-wall collisions [2] or fluctuations in the azimuthal electric field (Bohmtype diffusion) associated with fluctuations in the plasma density due to plasma turbulence [3]. Koo et al. investigated numerically two types of anomalous mechanisms for the UM/AFRL P5 HET and showed that the Bohm-type diffusion is preferable for the prediction of thruster performance while the electron-wall collision type is preferable for the estimation
This is an Open Access article distributed under the terms of the Creative Commons Attribution Non-Commercial License (http://creativecommons.org/licenses/by$\mathrm{nc} / 3.0 /$ which permits unrestricted non-commercial use, distribution, and reproduction in any medium, provided the original work is properly cited. cc * Associate Professor, Corresponding author : kybeom.kwon@gmail.com ** Associate Professor *** Professor 
of the potential profile [4]. Thus, Koo did not suggest a preference for one mechanism over the other. Boniface et al. studied anomalous electron diffusion experimentally and concluded that the electron-wall conductivity played a minor role in the anomalous transport [5]. These efforts indicate that while the anomalous electron cross field transport is not yet conclusively understood, Bohm-type diffusion is likely a major contributing factor. Research on anomalous electron diffusion continues with deeper physical investigations of the two mechanisms [6-10].

This study used a statistical approach in conjunction with a recently developed 1-D numerical method [11] to obtain a better understanding of the effects of anomalous electron diffusion. The method overcomes some of the deficiencies in self-consistency and difficulty in obtaining steady-state solutions for the variation of HET design parameters seen in the previously published models, while keeping the state-ofthe-art 1-D HET modeling. A brief summary of the method is described in the next section. Although the 1-D model cannot resolve the details of the HET physics, such as sheath phenomena and 3-D unsteady plasma properties, it can predict the thruster performance fairly well and efficiently from a computational point of view. Thus, this study was not intended to reveal the actual physical mechanisms that govern anomalous electron diffusion, but instead to investigate numerically the effects of anomalous electron diffusion on thruster performance and its relationship to important thruster parameters.

The numerical method developed includes Bohmtype diffusion as an additional collision event using the anomalous electron diffusion coefficients, which are still unknown here. Previous research uses the anomalous electron diffusion coefficient values that provide the best match with experimental results. This work treats the anomalous electron diffusion coefficients as free parameters and investigates their effects on the results by a numerical experiment through Design of Experiment (DOE). Furthermore, characteristics of the anomalous coefficient

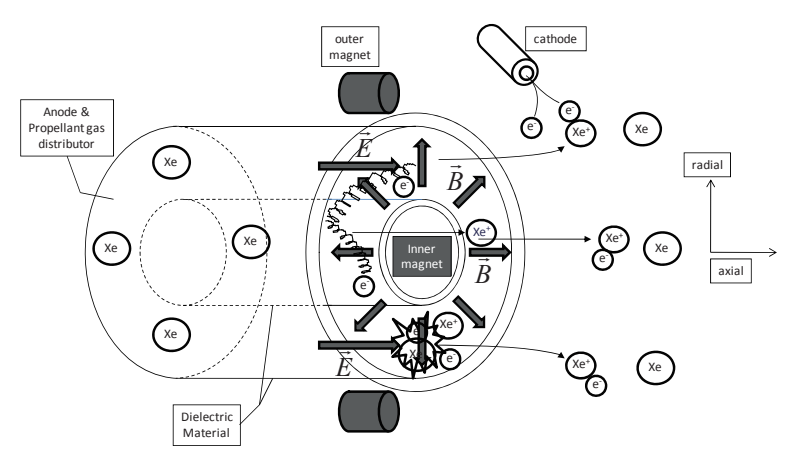

Fig. 1. Schematic of the HET variation are obtained indirectly by finding approximate values to match the experimental data. The results provide relationships between the electron anomalous diffusion, HET performance, and HET parameters. Additionally, the approach is used to find the radial magnetic field distribution required for a specific HET to reach given performance goals.

\section{The Numerical Method and Statistical Ap- proach}

\subsection{Physics Model}

Computational methods used to predict the behavior of the plasma discharge are divided into three categories: 1) kinetic modeling, 2) magnetohydrodynamic (MHD) modeling, and 3) hybrid modeling. The kinetic modeling method solves for individual particles, such as neutrals, ions, and electrons based on the Boltzmann equation [1214]. Although it can reveal detailed plasma properties, the computational cost is significant. MHD modeling focuses on the macroscopic plasma properties, which results in a relatively short calculation time $[15,16]$. Hybrid modeling is a mixed approach of the previous two methods. It treats the neutrals and ions as particles and the electrons as a fluid [17]. Although the computational cost is reduced compared with kinetic modeling, it is still computationally intensive.

To conduct a numerical experiment, this study uses a 1-D magnetohydrodynamic model to analyze the physics of the HET. 'One-dimensional' in this context means that the average plasma properties, such as plasma number density, electric potential, and electron velocity, are calculated based on the axial position from the anode to cathode, which includes regions inside and outside the thruster channel (Fig. 1). Because this work does not focus on the HET analysis model itself, a brief description about the model is given below. Ref. [11] provides the details of the method, numerical scheme, and modeling procedure. The method uses an iterative approach to obtain the solutions at steadystate HET operation. The method solves the MHD governing equations for neutral, ion, and electron species, which are properly fitted to the HET analysis. The analysis region is divided into three regions from the anode towards the cathode: 1) the anode region, 2) the presheath region, and 3) the ionization/acceleration region. The potential difference between the anode and the presheath edge is calculated as

$$
e \varphi_{\text {edge-d }}=k T_{e} \ln 0.5 \approx 0.6931 k T_{e}
$$

where $e$ is the electric charge, $\varphi_{\text {edge- } d}$ is the potential difference between the anode and the presheath edge, $k$ is 
the Boltzmann's constant, and $T_{e}$ is the electron temperature.

In the presheath region, the following governing equations are used.

$$
\frac{d \Gamma_{n}}{d z}=-S \quad \frac{d \Gamma_{i}}{d z}=S \quad \frac{d \Gamma_{e}}{d z}=S \quad \frac{d\left(n_{e} u_{i}^{2}\right)}{d z}=-\frac{e n_{e}}{m_{i}} \frac{d \varphi}{d z} \quad 0=-k T_{e} \frac{d n_{e}}{d z}+e n_{e} \frac{d \varphi}{d z}
$$

where, $\Gamma$ is the number flux of each species, $z$ is the thruster axial coordinate, $S$ is the ionization rate, $n$ is the particle number density, $\varphi$ is the electric potential, $u$ is the mean velocity of each species, and $m$ is the particle mass of each species.

The ionization/acceleration region uses the following governing equations.

$$
\begin{gathered}
u_{n} \frac{d n_{n}}{d z}=-S \quad \frac{d \Gamma_{i}}{d z}=S \quad \frac{d \Gamma_{e}}{d z}=S \\
n_{e}(z)=\int_{0}^{z} \frac{S^{\prime}\left(z^{\prime}\right) d z^{\prime}}{\left[u_{n, 0}^{2}+2 \frac{e}{m_{i}}\left(\varphi\left(z^{\prime}\right)-\varphi(z)\right)\right]^{1 / 2}}+\frac{n_{e, 0} u_{i, 0}}{\left[u_{n, 0}^{2}+2 \frac{e}{m_{i}}\left(\varphi_{0}-\varphi(z)\right)\right]^{1 / 2}} \\
\frac{d(e \varphi)}{d z}=\frac{m_{e} v_{e, m}}{n_{e}} \Gamma_{e} \quad \frac{d \varepsilon_{e}}{d z}=-\frac{3}{5} e E-\frac{3}{5} \frac{n_{e} v_{e, \varepsilon}}{\Gamma_{e}} \varepsilon_{e}-\frac{n_{e} v_{i}}{\Gamma_{e}} \varepsilon_{e}
\end{gathered}
$$

where, $v_{e, m}$ is the classical cross field electron momentum collision frequency, $v_{e, \varepsilon}$ is the electron energy loss collision frequency, $v_{i}$ is the ionization collision frequency, $\varepsilon_{e}$ is the electron energy, and subscript 0 represent the location where the ionization/acceleration region begins. Especially, $v_{e, m}$ and $v_{e, e f f}$ are calculated by the following.

$$
v_{e, m}=v_{e, e f f}\left(1+\frac{\omega_{e}^{2}}{v_{e, e f f}^{2}}\right), \quad v_{e, e f f}=v_{e n}+v_{e i}+v_{\text {wall }}+\alpha_{\text {ano }} \omega_{e}
$$

where, $v_{e, e f f}$ is the effective electron momentum collision frequency, $\omega_{e}$ is the electron cyclotron frequency, and $\alpha_{\text {ano }}$ is the anomalous coefficient. The term, $\alpha_{\text {ano }} \omega_{e}$ can be written as $v_{B o h m}$, which is the Bohm-type collision frequency. $\alpha_{a n o}$ is the input parameter used to artificially simulate the effect of fluctuations in the electric field. From the results of Ref. [5] and [18], the effects of the Bohm-type diffusion should change from inside the discharge channel to outside the discharge channel. In particular, the diffusion is approximately doubled outside the channel versus inside the channel. Thus, different values of the anomalous coefficients are used in this study according to the region.

In this method, the radial magnetic field distribution along the thruster axial coordinate up to the cathode is completely determined by three parameters, $B_{\text {max }}, B_{\text {cath }}$, and $K . B_{\max }$ is the maximum radial magnetic field strength, $B_{\text {cath }}$ is the radial magnetic field strength at the cathode, and $K$ is the magnetic field shape coefficient inside the channel. $B_{\max }$ and $K$ completely determine the distribution of the field magnitude inside the channel, given in the following analytical form as

$$
B(z)=B_{\max } \exp \left[-K\left(\frac{z}{L}-1\right)^{2}\right]
$$

$B_{\max }$ and $B_{\text {cath }}$ also determine the distribution outside the channel by the assumption of linear distribution. The point dividing the presheath region and the ionization/ acceleration region is called the matching point. The point is usually located near the anode, but it can be varied, based on the given input conditions such as thruster geometry, operating condition (mass flow rate, discharge voltage), and radial magnetic field distribution. Thus, location of the matching point must be solved self-consistently.

The inputs are the inner and outer radii of the thruster $\left(R_{1}, R_{2}\right)$, axial channel length $(L)$, the axial distance between channel exit and the cathode location $\left(z_{\text {cath }}\right)$, discharge voltage $\left(V_{d}\right)$, anode propellant mass flow rate $\left(\dot{m}_{a}\right)$, percentage of the propellant mass used for the cathode per anode mass flow rate $\left({ }_{p m c}\right)$, three radial magnetic field distribution parameters $\left(B_{\max }, B_{\text {cath }}, K\right)$, and two reciprocals of the anomalous coefficients inside and outside the channel $\left(\widehat{\alpha}_{\text {ano, }, \text { } n}, \widehat{\alpha}_{\text {ano,out }}\right)$. The outputs are the average axial plasma properties as a function of the $z$ coordinate from the anode to the cathode as well as the performance parameters, such as the thrust, specific impulse, efficiency, discharge current, and required power.

The method is numerically fast and robust for arbitrary input values, which has not been achieved with previous methods. Here 'robust' means that the method is not affected by the initial conditions and at least it gives the reason for the failed cases. However, the method cannot predict the discharge channel erosion rate and is only applicable for the HETs that have very low radial magnetic field strength near the anode.

\subsection{Classification of Solution}

To conduct numerical experiment for arbitrary variable ranges, solutions from the numerical method are classified. The method has a convergence criterion, i.e., if the normalized sum of relative changes in plasma properties $\left(\varepsilon_{e r r}\right.$ ) is less than the pre-specified tolerance $\left(\varepsilon_{t o l}\right)$, which is usually very small value normalized by the first error, for example, 1e-8, then the iteration stops and the plasma properties at that point in the simulation are taken as the solution. The method also employs the maximum iteration number $\left(N_{\max }\right)$ for the case of a non-convergent, but oscillatory solution.

1) Case I: $\varepsilon_{\text {err }} \leq \varepsilon_{\text {tol }}$ - Success

The solution procedure is considered successful and the converged solution is obtained. 
2) Case II: $\varepsilon_{e r r}>\varepsilon_{t o l}$ and $N_{\max }$ reached - Success

In this case, the solution procedure is also considered successful, but the error history shows oscillatory behavior. Although the relative error does not reach the pre-specified tolerance, the performance metrics show convergent behavior. Figures 2 and 3 show these characteristics. However, in this case we must take averages on performance parameters values during the last iteration steps, for example, $\sim 100$ iteration steps.

3) Case III: Low plasma number density at the matching point - Fail

The plasma number density at the matching point is updated at every iteration step. Initially, the plasma number density at the matching point is guessed as a low value, for example, $10^{16} \mathrm{~m}^{-3}$. If consecutive updates during the first iteration steps yield values lower than the first guessed value, two cases are observed. First, the plasma number density decreases initially and then increases over the first guessed value. The consequence of this process results in Case I or Case II, which is a successful solution. Second, the

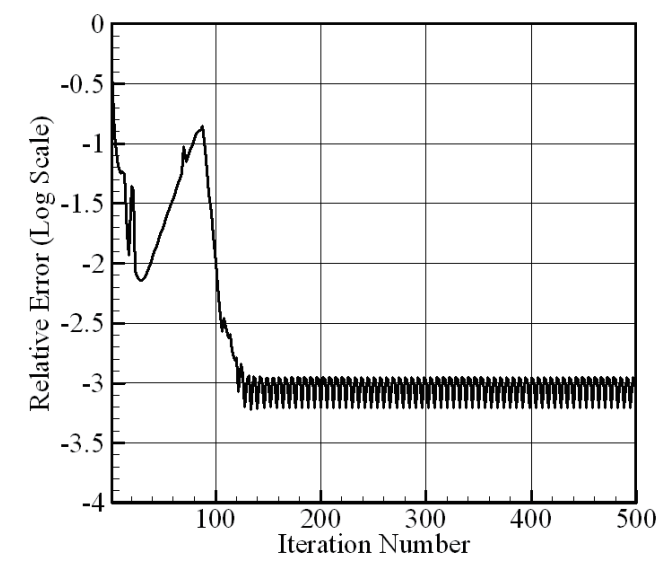

Fig. 2. Error behavior

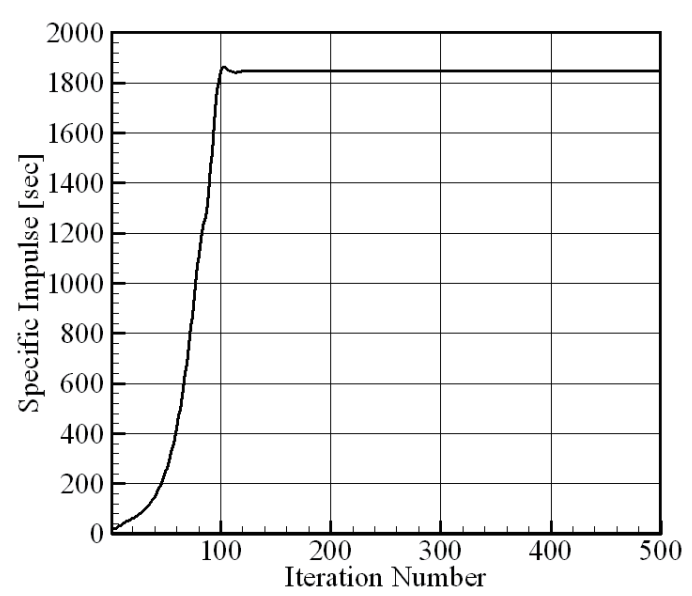

Fig. 3. Specific impulse convergence behavior plasma number density continues to decrease and reaches a negative value. This is physically impossible (failure case) and the solution process is ended. The reason for this seems to be due to low propellant mass flow rate, insufficient discharge voltage, or insufficient electron diffusion.

4) Case IV: Presheath region length < length of one computational grid cell - Fail

If the calculated presheath region length during iterations is less than the length of one computational grid cell, the solution process is ended. The cause of this is that the magnetic field strength in the region near the anode has attained a value that is too high, which is not common in the HET. Thus, this case is also classified as a failure case to avoid large magnitude magnetic field strength near the anode region.

5) Case V: Positive electron mean velocity at the cathode - Fail

In this case, the electron mean velocity at the cathode is positive during iteration steps, which means that electrons escape from the thruster. If this happens, the electrons cannot enter the channel and collide with the neutrals. There are multiple ways in which this failure case might occur. First, the thruster might require a higher potential difference between the anode and the cathode. Second, the electron diffusion may be insufficient due to a low electron temperature.

6) Case VI: Presheath region length $>$ thruster device length - Fail

The causes for this failure seem to be similar to those for Case V, especially that electron temperature at the matching point might have attained a value that is too low (cold plasma).

Based on observations of many numerical experiments on failure cases, Case III is the most common event, which clearly indicates that certain input combinations cannot achieve a plasma discharge for the given thruster. Thus, if this happens, it is very likely that the thruster could not be operated in view of 1-D modeling with the current numerical method. The physical connection between a successfully operating discharge and a successful simulation discussed here will be more explained in the next section.

\subsection{Statistical Approach}

This work used a statistical approach to study the effect of the anomalous electron diffusion in HET. The approach follows two steps: 1) perform DOE using the model with the given ranges of variables of interest, such as the anomalous coefficients, and 2) create response surface equations for the thruster performance parameters through regression. 
The response surface equation has the form

$$
y=\beta_{0}+\sum_{i=1}^{k} \beta_{i} x_{i}+\sum_{i=1}^{k} \beta_{i i} x_{i}^{2}+\sum_{i=1}^{k-1} \sum_{j=2}^{k} \beta_{i j} x_{i} x_{j}+\varepsilon
$$

where, $x_{1}, x_{2}, \cdots \cdots . ., x_{k}$ are the input variables, $y$ is the output variable, $\beta_{0}, \beta_{i i}(i=1, \cdots, k), \beta_{i j}(i=1, \cdots, k, j=1, \cdots, k)$ are the regression coefficients, and $\varepsilon$ is the random error.

Once the response surface equation is created, various statistical analyses can be made to investigate the effects of variables on thruster performance parameters for the given variable ranges. Furthermore, optimization based on the response surface equation can be done to find the values of variables for a certain objective (for example, finding values of the anomalous coefficients which best match the experimental values of performance parameters). This approach is used in Sections III through V.

\section{Study on the Effect of the Anomalous Electron Diffusion with the SPT-100 Ex- perimental Results}

\subsection{Numerical Exploration for Ranges of the Anoma- lous Coefficients}

The SPT-100 is a flight-proven HET [19]. The effect of the anomalous electron diffusion in the SPT-100 has been studied experimentally [20] and the specified ranges of the anomalous coefficients have been explored by numerical experimentation. As explained previously, researchers have used different values: Fife took the value of $\hat{\alpha}_{\text {ano,in }}$ (recall that $\hat{\alpha}$ is the reciprocal of the $\alpha$ ) as 107 [21], Ahedo et al. used the value of 100 [22], and Hofer et al. used 160 [23]. Thus, the selected range based on historical values of $\hat{\alpha}_{\text {ano,in }}$ is between 100 and 160. For $\hat{\alpha}_{\text {ano,out }}$, because the anomalous diffusion should be approximately doubled from the experimental results [5, 18], 50 and 80 are taken as the lower and upper bounds, respectively. The selected ranges of the anomalous coefficients will be fixed in this paper and the investigation of varying the ranges is left for future work. Numerical experiments were done with a six-level full factorial design (one of the DOE methods), which is a total of 36 runs.

Figure 4 shows grids that illustrate the success/fail cases of the solutions as well as the probability of success (Ps) for each mass flow rate case based on the classification given in the previous section. As the anode mass flow rate decreases, more failed cases are observed. Generally, solutions are obtained at higher values of the anode mass flow rate for the entire ranges of the anomalous coefficients. Thus, it can be seen that higher electron anomalous diffusion is required for the thruster to sustain discharge in the case of lower anode mass flow rate operation, which, in turn, indicates that if the anomalous electron diffusion is insufficient, then discharge cannot be established.

The effects of the anomalous coefficient variation on performance parameters are revealed through creating response surface equation using DOE results. The resulting regression coefficients, t-ratios, and p-values have the meaning of statistical significance of each anomalous electron diffusion coefficient on each response. The t-ratios and p-values are the results of a t-test in statistical analysis, which can be used to identify significance of the input parameters. For this work, the standard least-squares method in the JMP statistical software was used to create response surface models [24].

Table 1 shows the summary of fit for thrust at a $5.25 \mathrm{mg} / \mathrm{s}$ anode flow rate. Excellent goodness of fit was obtained, giving an almost-perfect regression. $R^{2}$ measures the proportion of the variation by fitting means to each factor level. The closer to 1 it is, the better the fit is. $R_{a d j}^{2}$ is almost the same measure as $R^{2}$, but it is a ratio of mean square error instead of the sums of the squares. Table 2 shows parameter estimates and the associated Pareto plot. The anomalous coefficient inside the channel plays a dominant $r$ ole in thrust variation. Although the effect of the square term of the inside anomalous coefficient is $10^{-3}$ times less, it is statistically significant, based on the p-value (Prob $>|t|)$. JMP uses the mark '*' in $\mathrm{p}$-value to indicate that the variable is statistically significant. The Pareto plot also indicates the relative significance of the variables.

The goodness of fit and parameter estimates for other performance parameters show a similar trend to the thrust, except for the total efficiency. Although the goodness of fit for

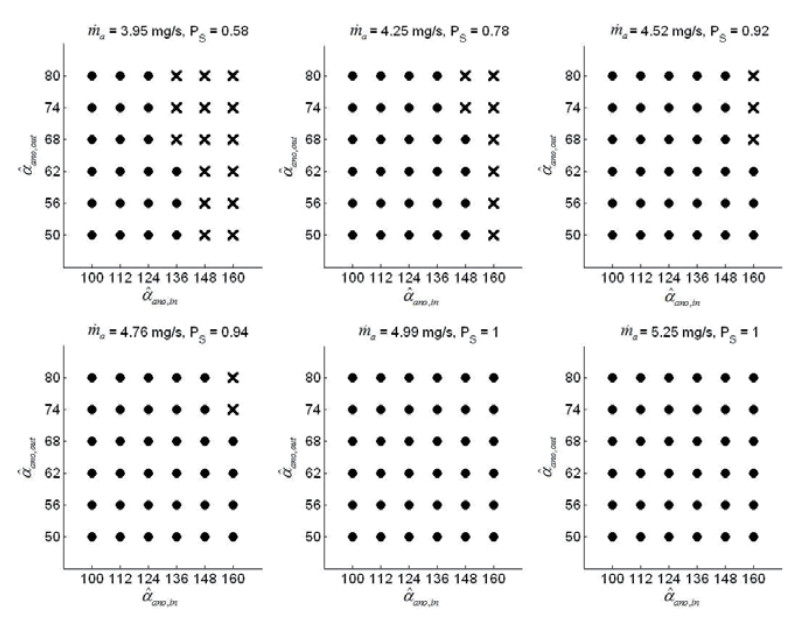

Fig. 4. Visualization of numerical experimentation (dot - success, cross - fail) 
the total efficiency is still high (Table 3), the trend of statistical significance of the parameters for the total efficiency is very different (Table 4). The square term of $\hat{\alpha}_{\text {ano,in }}$ has the highest absolute t-ratio value. However, all of the t-ratio values have a similar order of magnitude, except for the square term of $\hat{\alpha}_{\text {ano,out }}$. First, it can be implied that the anomalous electron diffusion coefficients alone are not reliable predictors of total efficiency. Second, the higher order or some nonlinear terms may be incorporated into the regression model. Third, the total efficiency should also be expressed by other internal parameters such as plasma density, electron energy, and the like. Note that the total efficiency in this study does not include the power used by the magnetic circuit and cathode heater.

Figure 5 shows the prediction profiler for the case of $\dot{m}_{a}=5.25 \mathrm{mg} / \mathrm{s}$. The profiler is generated based on the regression model in the range of each anomalous coefficient. It displays output profile traces for each variable. The trace is the predicted response as one variable is changed while the others are held constant at the present values. Thrust, discharge current, and total specific impulse show the same trends: as $\hat{\alpha}_{\text {ano, in }}$ and $\hat{\alpha}_{\text {ano,out }}$ increase, they decrease. Additionally, it can be identified that $\hat{\alpha}_{\text {ano,in }}$ has more of an impact on those responses than $\hat{\alpha}_{\text {ano,out }}$. The magnitude of anomalous electron diffusion is favorable to the thrust and the total specific impulse. The discharge current is simply proportional to the magnitude of anomalous electron diffusion. Because an increase in the discharge current results in an increase in total power, there should be a tradeoff between performance and power requirements, i.e., the thrust-to-power ratio.

The impact of the anomalous electron diffusion coefficients on the total efficiency is quite different from the other responses. First, the impact of $\hat{\alpha}_{\text {ano,out }}$ is opposite to that of the other performance metrics, i.e., as $\hat{\alpha}_{\text {ano,out }}$ increases, the total efficiency also increases. Furthermore, variation of the

Table 1. Summary of fit for thrust of the SPT-100

\begin{tabular}{cccc}
\hline \hline $\mathrm{R}^{2}$ & $\mathrm{R}_{\text {adj }}^{2}$ & Root Mean Square Error & Mean of Response \\
\hline 0.99864 & 0.998414 & 0.132092 & $95.2625 \mathrm{mN}$ \\
\hline \hline
\end{tabular}

Table 2. Parameter estimates and associated Pareto plot for thrust of the SPT-100

\begin{tabular}{|c|c|c|c|c|}
\hline Term & Estimate & t Ratio & $\operatorname{Prob}>|t|$ & Pareto Plot \\
\hline$\hat{\alpha}_{a n o, i n}$ & -0.158464 & -147.5 & $<.0001^{*}$ & \\
\hline$\hat{\alpha}_{\text {ano,out }}$ & -0.025595 & -11.91 & $<.0001 *$ & \\
\hline$\left(\hat{\alpha}_{a n o, i n}-130\right) \times\left(\hat{\alpha}_{a n o, i n}-130\right)$ & -0.000689 & -11.25 & $<.0001^{*}$ & I \\
\hline$\left(\hat{\alpha}_{\text {ano,in }}-130\right) \times\left(\hat{\alpha}_{\text {ano,out }}-65\right)$ & 0.0001951 & 1.86 & 0.0725 & 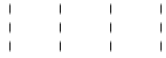 \\
\hline$\left(\hat{\alpha}_{\text {ano,out }}-65\right) \times\left(\hat{\alpha}_{\text {ano,out }}-65\right)$ & 0.0002232 & 0.91 & 0.3698 & 1 \\
\hline
\end{tabular}

Table 3. Summary of fit for total efficiency of the SPT-100

\begin{tabular}{cccc}
\hline $\mathrm{R}^{2}$ & $\mathrm{R}_{\text {adj }}^{2}$ & Root Mean Square Error & Mean of Response \\
\hline 0.982389 & 0.979454 & 0.000922 & 0.48969 \\
\hline \hline
\end{tabular}

Table 4. Parameter estimates and associated Pareto plot for total efficiency of the SPT-100

\begin{tabular}{|c|c|c|c|c|}
\hline Term & Estimate & t Ratio & Prob $>|t|$ & Pareto Plot \\
\hline$\left(\hat{\alpha}_{a n o, i n}-130\right) \times\left(\hat{\alpha}_{a n o, i n}-130\right)$ & $-1.335 \mathrm{e}-5$ & -31.22 & $<.0001^{*}$ & \\
\hline$\hat{\alpha}_{a n o, o u t}$ & 0.0003732 & 24.89 & $<.0001 *$ & \\
\hline$\left(\hat{\alpha}_{\text {ano,in }}-130\right) \times\left(\hat{\alpha}_{\text {ano,out }}-65\right)$ & $-5.867 e-6$ & -8.02 & $<.0001 *$ & \\
\hline$\hat{\alpha}_{a n o, i n}$ & $-2.854 \mathrm{e}-5$ & -3.81 & $0.0006^{*}$ & \\
\hline$\left(\hat{\alpha}_{\text {ano,out }}-65\right) \times\left(\hat{\alpha}_{\text {ano,out }}-65\right)$ & $-8.02 e-7$ & -0.47 & 0.6426 & 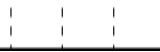 \\
\hline
\end{tabular}


total efficiency with $\hat{\alpha}_{\text {ano,in }}$ results in a quadratic shape. This indicates that there is an optimum magnitude of anomalous electron diffusion that yields the maximum allowable total efficiency. The reason for this phenomenon can be explained by examining the profiler for the anode efficiency and its component efficiencies. The anode efficiency does not include the mass flow rate used by the cathode and other powers used for the cathode operation and magnetic field generation.

Figure 6 shows the prediction profiler for the anode efficiency and two of the three component efficiencies. Electrical efficiency is the fraction of the ion beam current to total discharge current. Acceleration efficiency is a measure of the mean kinetic energy gained by the ions with the given electrical energy. Utilization efficiency is the fraction of the singly ionized beam flow rate to propellant mass flow rate. The utilization efficiency is nearly constant and has values close to 1 in most cases, indicating that most of the propellant neutral atoms are ionized. The effects of the anomalous electron diffusion coefficients on electrical and acceleration efficiencies are exactly opposite. A decrease in the electrical

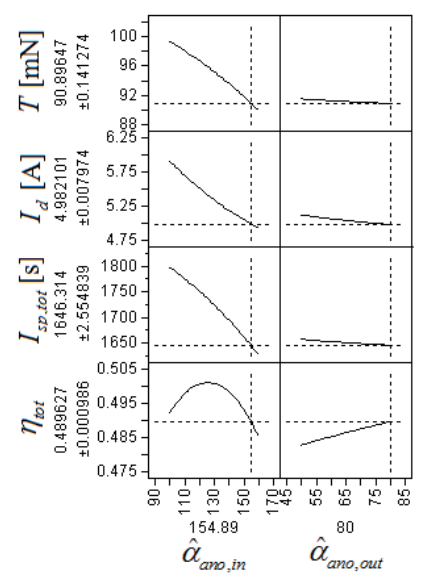

Fig. 5. Prediction profiler at an anode flow rate of $5.25 \mathrm{mg} / \mathrm{s}$ for the SPT-100

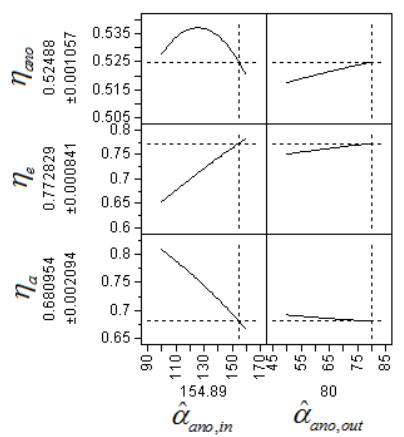

Fig. 6. Prediction profiler for efficiencies at $\dot{m}_{a}=5.25 \mathrm{mg} / \mathrm{s}$ for the SPT100 efficiency as the anomalous electron diffusion coefficients increase indicates that if the amount of anomalous electron diffusion increases, the ion beam current fraction of the discharge current decreases. However, ions in this case gain more mean kinetic energy at the given electrical energy, which results in increase of the acceleration efficiency. This may be due to the high electron current ionizing more neutrals at relatively closer locations to the anode. As a result, optimum efficiency exists based on the trade-off between these two component efficiencies in the view of the electron axial diffusion. Thus, for future HETs to have higher efficiencies, consideration on this trade-off may be key.

\subsection{Finding Correct Anomalous Coefficient Values}

To find the correct anomalous electron diffusion coefficient values for the SPT-100, the optimization was done with the response surface equations obtained in the previous section. Here, 'correct' means the anomalous electron diffusion coefficient values that best match the experimental performance values. The optimization task is to best match the values of the response surface equation with the experimental values, which lowers the sum of square errors between them. The parameter values of interest are thrust, discharge current, total specific impulse, and total efficiency. The desirability function in the prediction profiler of JMP was used for the optimization. In the desirability function, a user can specify the desirability for each variable, such as maximization, minimization, or a desired target value. The overall desirability is defined as the geometric mean of the desirability for each response. The optimization is performed internally when maximization of desirability is requested.

Figure 7 shows the prediction profiler with desirability function. The last column of the graph matrix is the

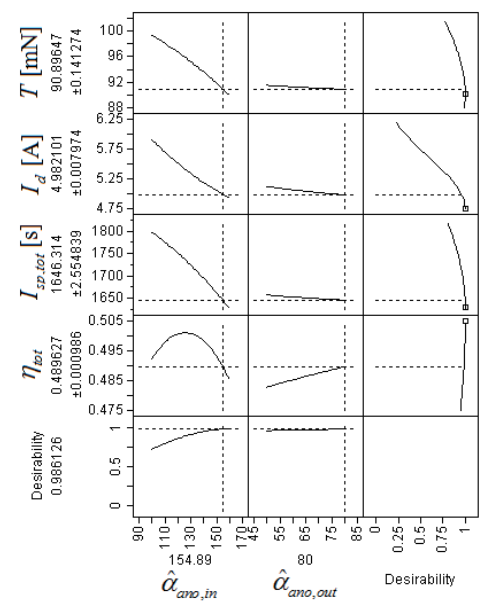

Fig. 7. Prediction profiler and desirability function at $\dot{m}_{a}=5.25 \mathrm{mg} / \mathrm{s}$ for the SPT-100 
desirability function for each response. Rectangles in the desirability function graphs indicate target values that need to be matched. The total efficiency shows relatively large deviations from the experimental values.

The correct anomalous electron diffusion coefficient values were found for all of the anode mass flow rate cases. An interesting result of this investigation was introduced in Ref. [11], which is repeated here in Figure 8. The inference is that the anomalous coefficient inside the channel and the cathode mass flow rate fraction are positively correlated. This means that the electron anomalous diffusion increases if the cathode mass flow rate fraction is increased, which yields higher electron axial conductivity.

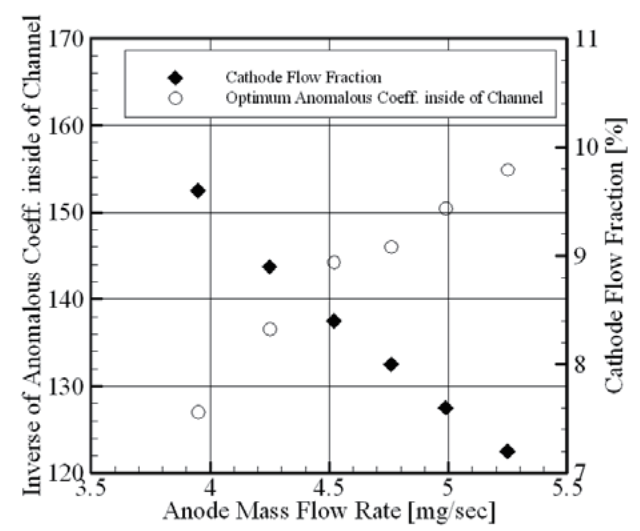

Fig. 8. Variation of $\hat{\alpha}_{\text {ano,in }}$ and cathode mass flow fraction with anode mass flow rate [11]

\section{Study on the Effect of the Anomalous Electron Diffusion with the High-Power HET Experimental Results}

For high-power HETs, the actual radial magnetic field distribution is not available due to restrictions imposed by the International Traffic in Arms Regulations (ITAR). However, the radial magnetic field distribution can be determined by extending the approach used for the SPT- 100 .

\subsection{Study with the T-220 HET}

The T-220 is a $10-\mathrm{kW}$ HET and its outer diameter is 220 $\mathrm{mm}$ [25]. The operating conditions and geometry are summarized in Table 5 .

To conduct the numerical experiment, the radial magnetic field distribution is required, which is not available. However, if the DOE is used, as in the previous section, an approximate radial magnetic field distribution can be obtained. The approach is a reverse engineering process.

It is evident that the number of numerical experiments increases because parameters characterizing the radial magnetic field distribution should be incorporated in the DOE. Thus, the variables in the DOE consist of three parameters for the magnetic field distribution and two parameters for the anomalous electron diffusion coefficients. The ranges of magnetic field variables are given in Table 6 .

In the actual numerical experiment, $\Delta B$, the difference between $B_{\max }$ and $B_{\text {cath }}$, is taken instead of $B_{\max }$ because $B_{\max }$ must be greater than $B_{c a t h}$, and it is preferable to have square space in terms of the DOE. The four-level full factorial design is created, which results in a total of 1024 runs. Because there are five variables $\left(\hat{\alpha}_{\text {ano, in }}, \hat{\alpha}_{\text {ano,out }}, \Delta B, B_{\text {cath }}, K\right)$ that have to be adjusted to match the experimental data, a rigorous optimization routine is applied to obtain accurate results.

The experimental data is taken from Ref. [26]. The results of the DOE are fed into the same statistical software to create the response surface equations. The goodness of fit for all of the responses is greater than 0.99 . In the process to find the optimum values, the normalized values, in terms of the median of each variable range, are used and the optimization performance index is chosen to be the sum of square of the

Table 5. T-220 operating conditions and geometry

\begin{tabular}{cccccccc}
\hline \hline & \multirow{2}{*}{$\dot{m}_{a}[\mathrm{mg} / \mathrm{s}]$} & $V_{d}[\mathrm{~V}]$ & $L$ & $R_{1}$ & $R_{2}$ & $W$ & $L_{\text {cath }}$ \\
\hline Design Point & 20 & 500 & 5 & 7.8 & 11 & 3.2 & 4 \\
Range & $15.7-22.1$ & $300-500$ & & & & & \\
\hline \hline
\end{tabular}

Table 6. Ranges of the magnetic field parameters for the T-220

\begin{tabular}{lcccc}
\hline & $B_{\max }[\mathrm{T}]$ & $\Delta B[\mathrm{~T}]$ & $B_{\text {cath }}[\mathrm{T}]$ & $K$ \\
\hline Lower Limit & 0.015 & 0.005 & 0.010 & 12 \\
Upper Limit & 0.031 & 0.013 & 0.018 & 20 \\
\hline
\end{tabular}


errors between the experimental data and the calculated value. Table 7 shows the results of the optimization results and a comparison with the experimental data and corresponding errors $(\leq 2 \%)$.

The inverse of the anomalous coefficient inside the channel reaches the lower bound. On the other hand, the inverse of anomalous coefficient outside the channel reaches the upper bound.

Figure 9 shows the prediction profiler and desirability function. The optimum values are applied for each parameter to investigate the design operation point of the T-220. $B_{\max }$ is the most significant factor, and $B_{\text {cath }}$ and $K$ have relatively minor effects on the variation of responses around the design point. The anomalous coefficients have relatively moderate effects. All of the parameters, except $K$, show the same trends of the effect, i.e., increasing $K$ values decreases the thrust, discharge current, total specific impulse, and total power, and results in increase in both efficiencies, but an increase in the other parameters shows the reverse effect.

The optimization results on the anomalous electron diffusion coefficients are still arbitrary in that an infinite number of combinations of the anomalous electron diffusion coefficients and the magnetic field parameters could exist for the given performance. Thus, further investigation

Table 7. Validation results for the T-220 design operating point

\begin{tabular}{cccc}
\hline \hline Parameters & Experimental Results & Numerical Results & Error (\%) \\
\hline$T[\mathrm{mN}]$ & 512 & 507 & -0.97 \\
$I_{\text {sp }, \text { tot }}[\mathrm{s}]$ & 2356 & 2348 & -0.34 \\
$\eta_{\text {ano }}[\%]$ & 65 & 64 & -1.54 \\
$\eta_{\text {tot }}[\%]$ & 57 & 56 & -1.75 \\
$I_{d}[\mathrm{~A}]$ & 20 & 20 & 0 \\
$P_{\text {tot }}[\mathrm{kW}]$ & 10.394 & 10.462 & +0.65 \\
$B_{\text {max }}[\mathrm{G}]$ & & $\mathbf{1 9 6}$ & \\
$B_{\text {cath }}[\mathrm{G}]$ & & $\mathbf{1 1 9}$ & \\
$K$ & & $\mathbf{1 2 . 1 2 8}$ & \\
$\hat{\alpha}_{\text {ano, in }}$ & & $\mathbf{1 0 0 . 0 0}$ & \\
$\hat{\alpha}_{\text {ano,out }}$ & & $\mathbf{8 0 . 0 0}$ & \\
\hline \hline
\end{tabular}

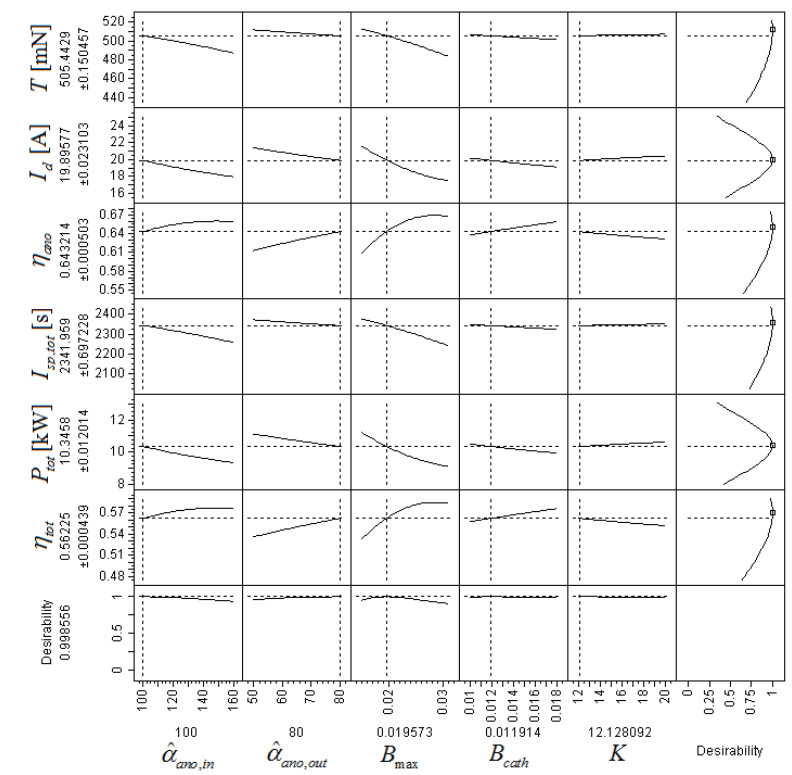

Fig. 9. Prediction profiler and desirability function for the T-220 on the effects of their variations is required to achieve a reliable approximate radial magnetic field distribution with quantitative confidence.

In preparation for this study, we investigated the relationship between axial electron diffusion and radial magnetic field distribution. For this purpose, a Monte Carlo (MC) method was used. Thus, $100 \times 100$ random values for the anomalous coefficients were generated over their ranges. The optimization routine was executed for each anomalous coefficient combination to obtain the optimum radial magnetic field distribution with the given anomalous coefficients. Note that the 'optimum' is defined as the parameters values that result in the closest match to the experimental data.

Figure 10 shows a contour of the optimization performance index. The minimum of the optimization performance index was at the point $\left(\widehat{\alpha}_{\text {ano,in }}, \hat{\alpha}_{\text {ano }, \text { out }}\right)=(100,80)$.

Figures 11 and 12 show the contours of $B_{\max }$ and $B_{\text {cath }}$, 


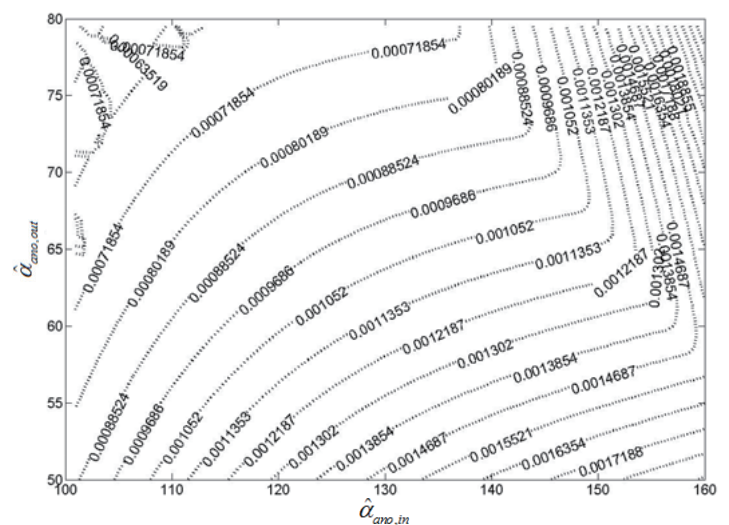

Fig. 10. Optimization performance index contour

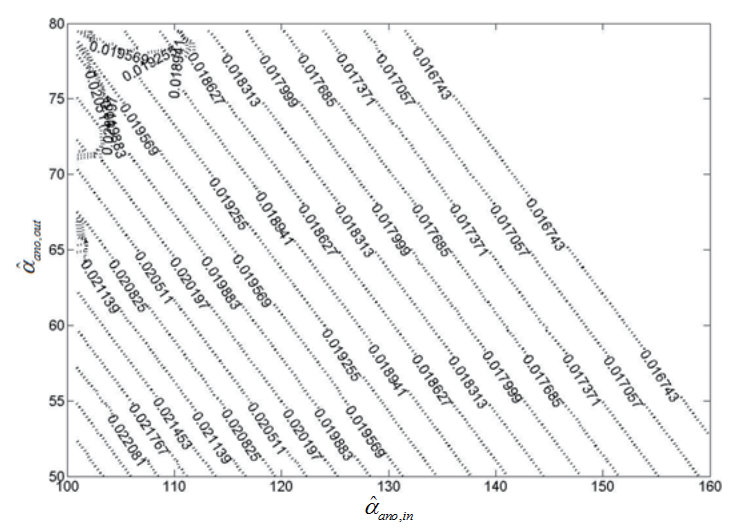

Fig. 11. Contour of $B_{\max }[T]$

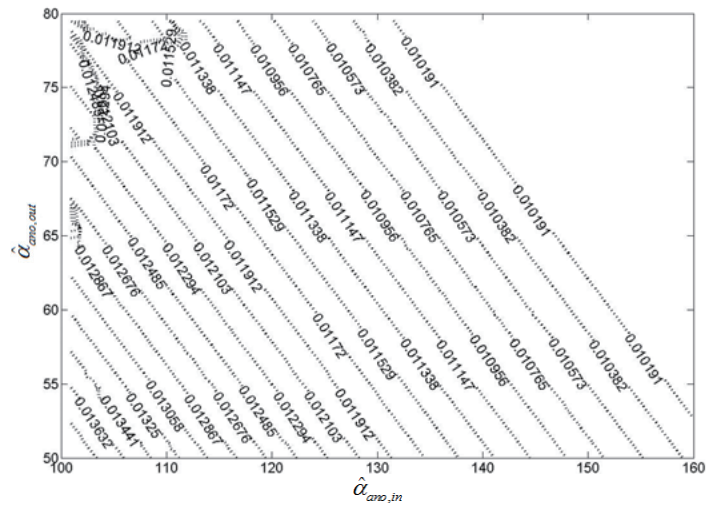

Fig. 12. Contour of $B_{\text {cath }}[\mathrm{T}]$ respectively. There is a near-linear relationship with the anomalous axial electron diffusion. This reveals that $B_{\max }$ and $B_{\text {cath }}$ should be increased when the anomalous axial electron diffusion is increased to match experimental data, i.e., if the anomalous electron diffusion increases, a large magnetic field strength is required to reduce axial electron mean velocity to maintain the same discharge current. Thus, the axial electron diffusion may be controlled by the magnetic field distribution. $K$ is not a significant factor compared with $B_{\max }$ and $B_{\text {cath }}$ for the T-220.

\subsection{Study with the NASA-457M HET}

The NASA-457M HET is a $50-\mathrm{kW}$ class HET. The outer diameter of the NASA-457M discharge channel is $457 \mathrm{~mm}$. Execution of the numerical experiment with experimental data obtained from the NASA-457M is more difficult because the dimensions of the discharge channel and radial magnetic field distribution are not available in the public literature. Based on the outer diameter of the NASA-457M, the other dimensions were determined from Figure 1 of Ref. [27] and are presented in Table 8.

The variables and their ranges for the DOE are the same as those for the T-220. However, in this case, based on the DOE experiences from the SPT-100 and the T-220, another DOE design strategy was used to reduce the DOE run time, which was a combination of the Central Composite Design (CCD) and the Latin Hypercube Sampling (LHS). It should be noted that the purpose of DOE is to acquire the maximum amount of information from the data with the least number of experimental runs. Figure 13 shows their schematics.

The advantage of CCD is that is covers the extremes of the concerned space and minimizes the extrapolation. However,

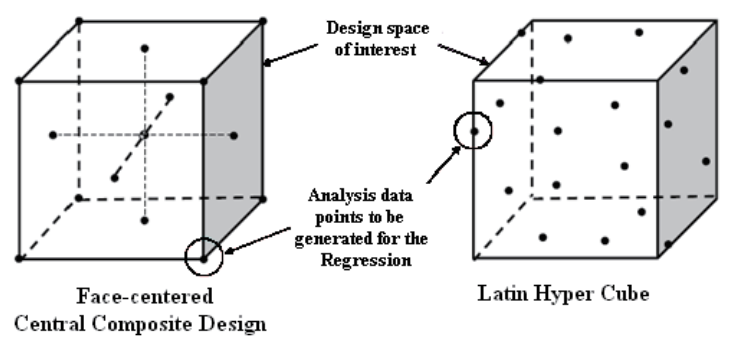

Fig. 13. Schematics of CCD and LHS

Table 8. NASA-457M operating conditions and estimated geometry

\begin{tabular}{cccccccc}
\hline \hline & \multirow{2}{*}{$\dot{m}_{a}[\mathrm{mg} / \mathrm{s}]$} & $V_{d}[\mathrm{~V}]$ & \multicolumn{5}{c}{ Estimated Geometry [cm] } \\
& & $L$ & $R_{1}$ & $R_{2}$ & $W$ & $L_{\text {cath }}$ \\
\hline Range & $15 \sim 93$ & $300 \sim 650$ & 5.9354 & 16.56 & 22.85 & 6.29 & 2.284 \\
\hline \hline
\end{tabular}


as the size of the space becomes large, many experimental cases might not have converged solutions due to the emphasis on the extremes. On the other hand, the LHS has rich samplings within the interior of the space. As a result, higher accuracy can be obtained on the interior design space. This advantage of LHS reduces the accuracy on the edges of the space. Thus, a combination of the two design methods is expected to yield a reasonable DOE table. For the given five variables, the CCD produces 43 runs. An additional 157 runs are created by the LHS, making a total of 200 runs.

The experimental data were taken from Ref. [27]. The experimental data were given by varying the anode mass flow rate from 15 to $93 \mathrm{mg} / \mathrm{s}$ and the discharge voltage from 300 to $650 \mathrm{~V}$ over a range of input powers from 9 to $72 \mathrm{~kW}$. Among these data, four experimental points were selected for the present study. The anode mass flow rate and the cathode mass flow rate for all points were $74.3 \mathrm{mg} / \mathrm{s}$ and 7.5 $\mathrm{mg} / \mathrm{s}$, respectively. The resulting percentage of the cathode mass flow rate (pmc) was then calculated as $10.09 \%$.

The results of the DOE for the discharge voltage of 300 $\mathrm{V}$ show 116 failure cases out of 200 total cases. For the discharge voltage of $402 \mathrm{~V}$, only four failure cases occur. The DOE for the other two discharge voltage cases, of $500 \mathrm{~V}$ and $649 \mathrm{~V}$, had no failure cases. This somewhat coincides with the results for the SPT-100. For the present case, a low discharge voltage caused a large number of failure cases. This reveals that the discharge voltage is also related to the anomalous electron diffusion, i.e., high discharge voltage could sustain the discharge with the large variation of the anomalous electron diffusion.

Although the number of runs is reduced for each case compared with the T-220 cases, the goodness of fit for all cases from regression results was above an $\mathrm{R}^{2}$ value of 0.99. The same procedure to find the optimum solutions for the anomalous electron diffusion coefficients and the magnetic field distribution parameters was applied for each discharge voltage validation point to investigate variations of magnetic field distribution with the discharge voltage. Figures 14 and Fig. 15 show the results. The performance metrics calculated with the correct values show great accuracy when compared with the experimental data for all discharge voltage points. The maximum magnetic field strength and the cathode magnetic field strength tend to increase as the discharge voltage increases. A large potential drop between the anode and the cathode entails a relatively high electric field. This, in turn, requires a higher radial magnetic field strength, enough to trap the electrons for the given performance capability. The anomalous electron diffusion coefficient outside the channel is nearly constant. The anomalous electron diffusion coefficient inside the channel decreases when the discharge voltage increases, except for the case of $500 \mathrm{~V}$. This may partially indicate that higher anomalous electron diffusion is required at lower discharge voltage to sustain discharge.

Because the anomalous electron diffusion coefficients are arbitrarily obtained from the optimization process, their values only hold for each discharge voltage. Furthermore, as seen in the T-220 validation results, there are an infinite number of solutions when considering the trade-offs between the anomalous electron diffusion coefficients and the magnetic field distribution parameters for given performance data. Although the same procedure can be applied as done in the T-220 case, and the resulting magnetic field distribution for each discharge voltage case can be accepted with certain confidence; it is worth taking a more rigorous analysis when considering the fact that the same magnetic field distribution is usually used for a specific thruster. This can also be deduced from the experimental data. The power required for the magnetic
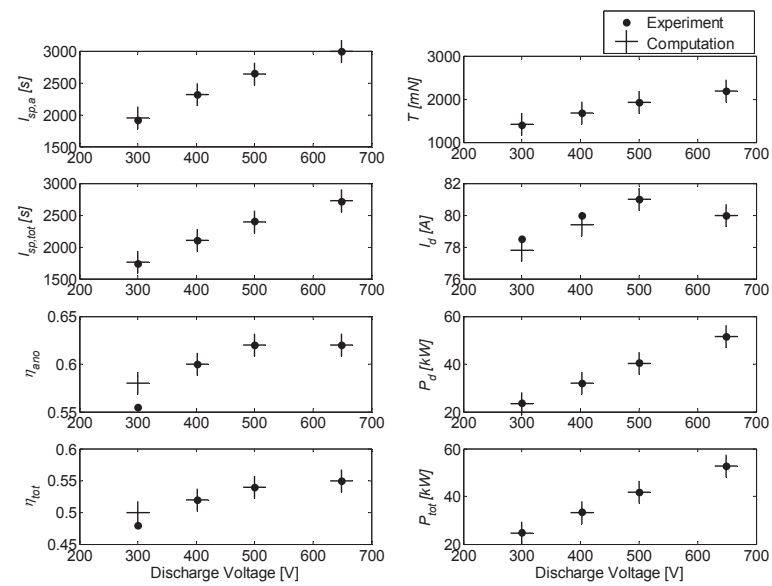

Fig. 14. Performance validation of the NASA-457M
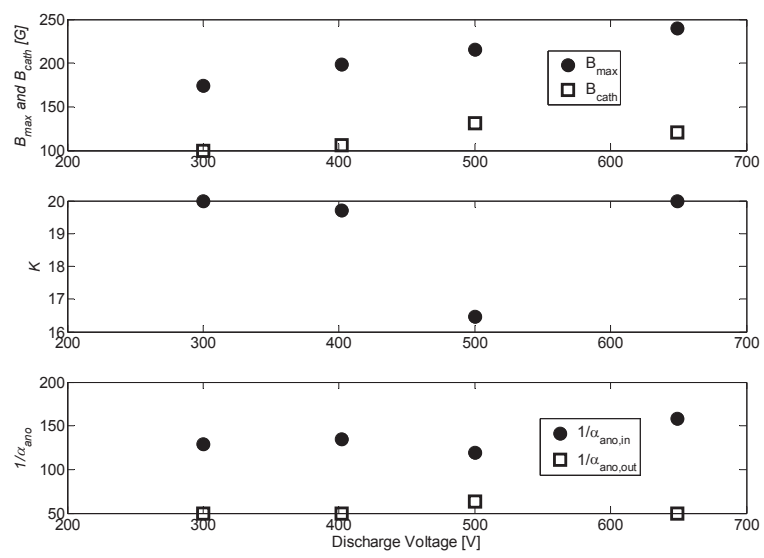

Fig. 15. Variations of optimum magnetic parameters and anomalous coefficients 
field generation and the cathode operation are calculated as $1.196,1.355,1.302$, and $1.213 \mathrm{~kW}$ for $300,402,500$, and $649 \mathrm{~V}$, respectively. It is clear that there is little difference for electric magnet power consumptions with the same cathode mass flow rate for each voltage case. Thus, all four voltage cases require similar magnetic field distributions. In the optimization process, this can be done by solving one optimization problem with three global variables for magnetic field distribution and eight anomalous coefficients.

The performance index is expanded to match all metrics for all voltage cases. Figures 16 and 17 show the results of this magnetic field optimization strategy.

The accuracy on the performance metrics is still high. Based on variation in the anomalous electron diffusion
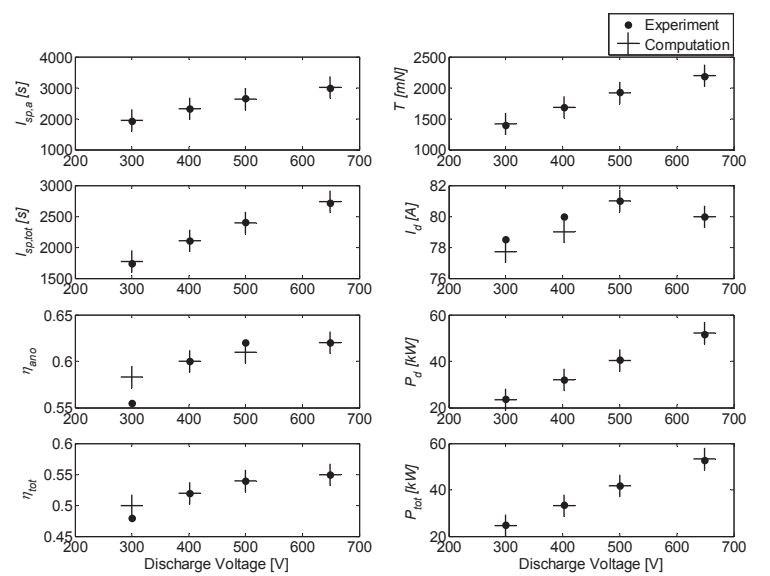

Fig. 16. Performance validation of the NASA-457M with one magnetic field distribution

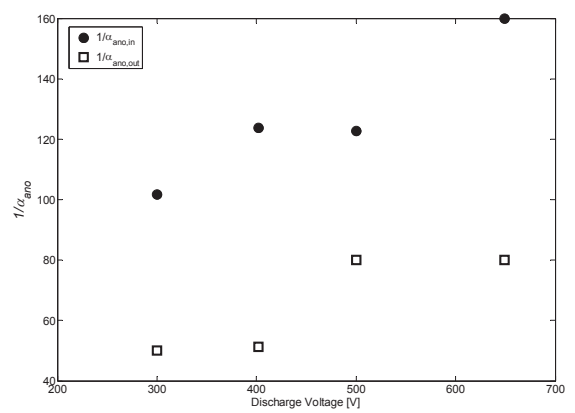

Fig. 17. Variations of anomalous coefficients for one magnetic field distribution coefficient, it is likely that higher anomalous electron diffusion occurs as the discharge voltage decreases when the same magnetic field distribution is applied for the thruster. This indicates that higher discharge voltage has a negative impact on the anomalous electron diffusion, as was observed in Ref. [5] where the anomalous coefficient increased as the discharge voltage decreased below $400 \mathrm{~V}$.

\section{Approximation of Radial Magnetic Field Distribution with Given Performance Goals}

This section discusses how the radial magnetic field distribution is approximated with the statistical approach in the previous sections when performance goals of a specific HET are given. To show this capability, the P5 HET is considered. The detailed operating conditions and geometry found in the literature are summarized in Table 9 [28].

Because the radial magnetic field distribution of the P5 is not restricted by the ITAR regulations, the exact magnetic field distribution is available [29]. However, the actual magnetic field distribution has relatively high strength near the anode region, which is not good because ions can be created near the anode region and converted back to neutrals due to wall collisions before exiting the channel.

To create a better radial magnetic field distribution, a situation is assumed such that there is a given HET geometry and there are also performance goals required for the specific HET. The question is what radial magnetic field distribution is required to produce those performance goals. Thus, the objective is to find the required $B_{\max }, B_{\text {cath }}$, and $K$.

The P5 is the given thruster and the experimental data of its performance metrics become performance goals. If the same method used for the T-220 is used, the required radial magnetic field distribution parameters are expected to be found. Since the anomalous coefficients are still arbitrary, those are included as before. Table 10 shows the ranges for each variable are selected based on the power level of the P5.

A four-level factorial design is created for $B_{\max }, B_{\text {cath }}, K$, $\hat{\alpha}_{\text {ano,in }}$, and $\hat{\alpha}_{\text {ano,out }}$. Thus, in total, 1024 runs were executed and

Table 9. P5 operating conditions and geometry

\begin{tabular}{cccccccc}
\hline \hline & \multirow{2}{*}{$\dot{m}_{a}[\mathrm{mg} / \mathrm{s}]$} & $V_{d}[\mathrm{~V}]$ & \multicolumn{5}{c}{ Geometry [cm] } \\
& & & $L$ & $R_{1}$ & $R_{2}$ & $W$ & $L_{\text {cath }}$ \\
\hline Design Point & 10.248 & 300 & & & & & \\
Range & $5.66 \sim 10.25$ & $200 \sim 500$ & 3.810 & 6.096 & 8.636 & 2.540 & 3 \\
\hline \hline
\end{tabular}


those are found with the same method. Table 11 shows the results. The results of performance metrics calculated from the optimization are very close compared with experimental performance data with the experimental uncertainty. The errors of the experimental data were $+1.2 /-8.0 \mathrm{mN}$ for the thrust, $+11 /-75$ s for the specific impulse, and $+0.7 /-4.9 \%$ for the anode efficiency. Bmax was calculated as 120 Gauss, which is slightly higher than the actual one.

Figure 18 shows the actual and candidate radial magnetic field distributions. The candidate distribution has lower magnetic field strength in most of the region inside the channel and a higher magnetic field strength in other regions than the actual P5. Note, at the time of the development of P5, the technology for lowering the magnetic field near the anode was not completely understood. Higher magnetic strength near the anode causes a negative effect on ions that could return to neutrals once ionized. Thus, if the candidate magnetic field is used today, the same performance could be guaranteed with less of a drawback. That is, if one were to design a new HET for the given performance goals from certain space mission requirements, the required radial magnetic field strength can be estimated using this approach, which is expected to provide better performance compared with one with higher radial magnetic field strength near the anode. The results of this study show that the approach developed can be used quite effectively to obtain an approximate radial magnetic field distribution for a specific thruster geometry to achieve the given performance goals.

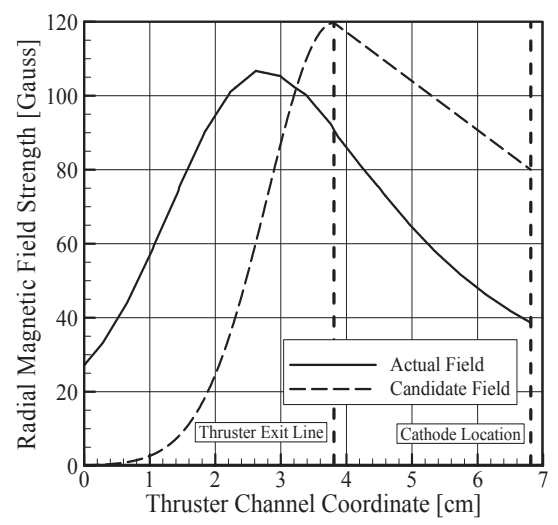

Fig. 18. Actual and candidate radial magnetic field distribution of the P5

Table 10. Ranges of magnetic field parameters for the P5

\begin{tabular}{lcccc}
\hline \hline & $B_{\max }[\mathrm{T}]$ & $\Delta B[\mathrm{~T}]$ & $B_{\text {cath }}[\mathrm{T}]$ & $K$ \\
\hline Lower Limit & 0.005 & 0.005 & 0.002 & 1 \\
Upper Limit & 0.015 & 0.013 & 0.008 & 16 \\
\hline \hline
\end{tabular}

Table 11. Results of finding candidate radial magnetic field distribution for the P5

\begin{tabular}{cccc}
\hline \hline Parameters & Experimental Results & Numerical Results & Error (\%) \\
\hline$T[\mathrm{mN}]$ & 178 & 180 & +1.12 \\
$I_{\text {sp tot }}[\mathrm{s}]$ & 1683 & 1696 & +0.77 \\
$\eta_{\text {ano }}[\%]$ & 51 & 50 & -1.96 \\
$\eta_{\text {tot }}[\%]$ & - & 47 & - \\
$I_{d}[\mathrm{~A}]$ & 10.4 & 10.6 & +1.92 \\
$P_{\text {tot }}[\mathrm{kW}]$ & 3.12 & 3.18 & +1.92 \\
$B_{\text {max }}[\mathrm{G}]$ & & $\mathbf{1 2 0}$ & \\
$B_{\text {cath }}[\mathrm{G}]$ & & $\mathbf{8 0}$ & \\
$K$ & & $\mathbf{7 . 0 1}$ & \\
$\hat{\alpha}_{\text {ano, in }}$ & & $\mathbf{1 1 4 . 2 6}$ & \\
$\hat{\alpha}_{\text {ano out }}$ & & $\mathbf{7 9 . 4 8}$ & \\
\hline \hline
\end{tabular}




\section{Conclusions}

Although many efforts have been made to reveal the physical mechanisms that govern the phenomenon of anomalous electron diffusion, it is still not clearly understood. This study used a numerical method to improve our understanding of the effects anomalous electron diffusion on thruster performance and the relationship with thruster design parameters. First, an increase in the cathode mass flow rate fraction, i.e., increased number of electrons generated by the cathode, can increase the axial electron diffusion. Second, the amount of the axial electron diffusion inside the channel dominates the thrust variation for a given thruster. Third, the effects of axial electron diffusion on electrical and acceleration efficiencies tend to be opposite, meaning that an increase in the anomalous electron diffusion lowers the ion beam current fraction of the discharge current, but boosts the ion mean kinetic energy instead. Thus, to achieve maximum HET efficiency requires a trade-off with values of the anomalous electron diffusion. Furthermore, the approach can be used to determine the required radial magnetic field distribution to achieve given performance goals of a specific thruster for future space missions.

For future work, based on understanding on the electron anomalous diffusion and proper range identification of the anomalous diffusion coefficient values with the existing HETs in this study, a HET design environment will be developed using a probabilistic design approach for the uncertain anomalous coefficients.

\section{References}

[1] Oh, D. Y., "Evaluation of solar electric propulsion technologies for discovery class missions", Journal of Propulsion and Power, Vol. 44, 2007, pp. 399-411.

[2] Morozov, A., Esipchuk, Y., Tilinin, G., Trofimov, A., Sharov, Y. and Shchepkin, G. Y., "Plasma accelerator with closed electron drift and extended acceleration zone", Soviet Physics - Techincal Physics, Vol. 17, 1972, pp. 38-45.

[3] Janes, G. and Lowder, R., "Anomalous electron diffusion and ion acceleration in a low-density plasma", Physics of Plasmas, Vol. 9, 1966, pp. 1115-1123.

[4] Koo, J. W. and Boyd, I. D., "Computational model of a Hall thruster", Computer Physics Communications, Vol. 164, 2004, pp. 442-447.

[5] Boniface, C., Garrigues, L., Hagelaar, G. J. M., Boeuf, J. P., Gawron, D. and Mazouffre, S., "Anomalous cross field electron transport in a Hall effect thruster", Applied Physics
Letter, Vol. 89, 2006, 161503.

[6] Bultinck, E., Mahieu, S., Depla, D. and Bogaerts, A., "The origin of Bohm diffusion, investigated by a comparison of different modeling methods", Journal of Physics D: Applied Physics, Vol. 43, 2010, 292001.

[7] Li, H., Zhang, F., Liu, H. and Yu, D., "Characteristics of electron near-wall transport under two-dimensional dynamic sheath in a Hall effect thruster", Physics of Plasmas, Vol. 17, 2010, 074505.

[8] Raitses, Y., Kaganovich, I. D., Khrabrov, A., Sydorenko, D., Fisch, N. J. and Smolyakov, A., "Effect of secondary electron emission on electron cross-field current in $\mathrm{E} \times \mathrm{B}$ discharges", IEEE Transaction on Plasma Science, Vol. 39, 2011, pp. 995-1006.

[9] Hagelaar, G. J. M. and Oudini, N., "Plasma transport across magnetic field lines in low-temperature plasma sources", Plasma Physics and Controlled Fusion, Vol. 53, 2011, 124032 .

[10] Coche, P. and Garrigues, L., "Study of stochastic effects in a Hall effect thruster using a test particles MonteCarlo model", Proceedings of the $32^{\text {nd }}$ International Electric Propulsion Conference, IEPC-2011-255, Wiesbaden, Germany, September, 2011.

[11] Kwon, K., Walker, M. L. R. and Mavris, D. N., "Selfconsistent, one-dimensional analysis of the Hall effect thruster", Plasma Sources Science and Technology, Vol. 20, 2011, 045021.

[12] Roy, R. I. S., Hastings, D. E. and Taylor, S., "Threedimensional plasma particle-in-cell calculations of ion thruster backflow contamination", Journal of Computational Physics, Vol. 128, 1996, pp. 6-18.

[13] Szabo, J. J., "Fully kinetic numerical modeling of a plasma thruster", Ph.D. Thesis, Aeronautics and Astronautics, Massachusetts Institute of Technology, 2001.

[14] Adam, J. C., Heron, A. and Laval, G., "Study of stationary plasma thrusters using two-dimensional fully kinetic simulations", Physics of Plasmas, Vol. 12, 2004, pp. 295-305.

[15] Toth, G., Ma, Y. and Gombosi, T. I., "Hall magnetohydrodynamic on block-adaptive grids", Journal of Computational Physics, Vol. 227, 2008, pp. 6967-6984.

[16] Ahedo, E., Cerezo, P. M. and Martinez-Sanchez, M., "One-dimensional model of the plasma flow in a Hall thruster", Physics of Plasmas, Vol. 8, 2001, pp. 3058-3068.

[17] Fife, J. M., "Hybrid-PIC modeling and electrostatic probe survey of Hall thruster", Ph.D. Thesis, Aeronautics and Astronautics, Massachusetts Institute of Technology, 1998.

[18] Koo, J. W. and Boyd, I. D., "Modeling of anomalous electron mobility in Hall thrusters", Physics of Plasmas, Vol. 13, 2006, 033501 . 
[19] Clauss, C. W., Tilley, D. L. and Barnhart, D. A., "Benefits of low-power stationary plasma thruster propulsion for small satellites", Proceedings of the $9^{\text {th }}$ AIAA/USU Conference on Small Satellites, Logan, Utah, 1995.

[20] Sankovic, J. M., Manley, J. A. and Haag, T. W., "Performance evaluation of the Russian SPT-100 thruster at NASA LeRC", Proceedings of the $23^{\text {rd }}$ International Electric Propulsion Conference, IEPC-93-094, Seattle, Washington, September, 1993.

[21] Fife, J. M., “Two-dimensional hybrid particle-in-cell modeling of Hall thruster", Master Thesis, Aeronautics and Astronautics, Massachusetts Institute of Technology, 1995.

[22] Ahedo, E. Gallardo, J. M. and Martinez-Sanchez, M., "Model of the plasma discharge in a Hall thruster with heat conduction", Physics of Plasmas, Vol. 10, 2002, pp. 3397-3409.

[23] Hofer, R. R., Katz, I. Mikellides, I. G. and GameroCastano, M., "Heavy particle velocity and electron mobility modeling in hybrid-PIC Hall thruster simulations", Proceedings of the 42nd AIAA/ASME/SAE/ASEE Joint Propulsion Conference \& Exhibit, AIAA-2006-4658, Sacramento, California, July 2006.

[24] SAS Institute Inc., “JMP 8.0 Help File”, 2008.
[25] Mason, L. S., Jankovsky, R. S. and Manzella, D. H., "1000 hours of testing on a 10 kilowatt Hall effect thruster", Proceedings of the $37 t^{h}$ AIAA/ASME/SAE/ASEE Joint Propulsion Conference, AIAA-2001-3773, Salt Lake City, Utah, July 2001.

[26] Jankovsky, R. S. and McLean, C., "Preliminary evaluation of a $10 \mathrm{~kW}$ Hall thruster", Proceedings of the $37^{\text {th }}$ AIAA Aerospace Sciences Meeting and Exhibit, AIAA-1999456, Reno, Nevada, January 1999.

[27] Manzella, D., Jankovsky, R. and Hofer, R., "Laboratory model $50 \mathrm{~kW}$ Hall thruster", Proceedings of the $38^{\text {th }}$ AIAA/ ASME/SAE/ASEE Joint Propulsion Conference \& Exhibit, AIAA-2002-3676, Indianapolis, Indiana, July 2002.

[28] Haas, J. M., Gulczinski, F. S., Gallimore, A. D., Spanjers, G. G. and Spores, R. A., "Performance characteristics of a 5 $\mathrm{kW}$ laboratory Hall thruster", Proceedings of the $34^{\text {th }}$ AIAA/ ASME/SAE/ASEE Joint Propulsion Conference \& Exhibit, AIAA-1998-3503, Cleveland, Ohio, July 1998.

[29] Gulczinski, F. S., "Examination of the structure and evolution of ion energy properties of a $5 \mathrm{~kW}$ class laboratory Hall effect thruster at various operational conditions", Ph.D. Thesis, Aerospace, The University of Michigan, 1999. 